\title{
BMJ Open Contemporary trends in global mortality of sepsis among young infants less than 90 days old: protocol for a systematic review and meta-analysis
}

\author{
Jen Heng Pek (D) , ${ }^{1}$ Ming Ying Gan, ${ }^{2}$ Bei Jun Yap, ${ }^{2}$ Shu Ting Tammie Seethor, ${ }^{2}$ \\ Rachel G Greenberg, ${ }^{3}$ Christoph Paul Vincent Hornik, ${ }^{4}$ Bobby Tan, ${ }^{5}$ Jan Hau Lee, ${ }^{6}$ \\ Shu-Ling Chong (iD ${ }^{7}$
}

To cite: Pek JH, Gan MY, Yap BJ, et al. Contemporary trends in global mortality of sepsis among young infants less than 90 days old: protocol for a systematic review and meta-analysis. BMJ Open 2020;10:e038815. doi:10.1136/ bmjopen-2020-038815

- Prepublication history and additional material for this paper are available online. To view these files, please visit the journal online (http://dx.doi. org/10.1136/bmjopen-2020038815).

Received 25 March 2020 Revised 24 June 2020

Accepted 25 June 2020
Check for updates

(C) Author(s) (or their employer(s)) 2020. Re-use permitted under CC BY-NC. No commercial re-use. See rights and permissions. Published by BMJ.

For numbered affiliations see end of article.

Correspondence to Dr Shu-Ling Chong; chong.shu-ling@kkh.com.sg

\section{ABSTRACT}

Introduction Neonatal sepsis has a high mortality rate that varies across different populations. We aim to perform a contemporary global evidence synthesis to determine the case fatality rates of neonatal sepsis, in order to better delineate this public health urgency and inform strategies to reduce fatality in this high-risk population.

Methods and analysis We will search PubMed, Cochrane Central, Embase and Web of Science for articles in English language published between January 2010 and December 2019. All clinical trials and observational studies involving infants less than 90 days old with a clinical diagnosis of sepsis and reported case fatality rate will be included. Two independent reviewers will screen the studies and extract data on study variables chosen a priori. Quality of evidence and risk of bias will be assessed using Cochrane Collaboration's tool and ROBINS-I. Results will be synthesised qualitatively and pooled for meta-analysis. Ethics and dissemination No formal ethical approval is required as there is no collection of primary data. This systematic review and meta-analysis will be disseminated through conference meetings and peer-reviewed publications.

PROSPERO registration number CRD42020164321.

\section{INTRODUCTION}

Neonatal sepsis accounts for more than 1.4 million deaths worldwide, with varying mortality in different geographical locations. ${ }^{1-3}$ It is a systemic condition of bacterial, viral or fungal origin with an array of clinical manifestations. ${ }^{45}$ Neonates exposed to these pathogens during the perinatal period are susceptible to invasive infections due to their relatively compromised immune system. ${ }^{1}$ Despite recent advances in diagnostic, treatment and preventive strategies, neonatal sepsis is still a leading cause of death in this population, with 4 out of every 10 neonates with sepsis dying or experiencing significant morbidity. ${ }^{267}$ Among these, premature neonates have the worst outcomes. ${ }^{8}$

\section{Strengths and limitations of this study}

- This review will synthesise the association and temporal trends between neonatal sepsis and death over the past decade.

- Analysis of subgroups will provide insights about mortality across gestation age, birth weight, timing of sepsis, setting where sepsis was acquired and geographical location.

- This protocol adheres to Preferred Reporting Items for Systematic Reviews and Meta-Analyses guidelines to ensure objectivity in literature search, data synthesis and reporting of outcomes.

- Heterogeneity across studies may exist due to differences in patient populations, settings and studies performed over a decade.

- The inclusion of only studies published in English may lead to a loss of data published in other languages.

Understanding worldwide mortality data on neonatal sepsis can provide valuable information to inform interventions in specific geographical locations. Mortality rates will differ due to the prevalence of pathogens, neonatal and maternal risk factors, and accessibility to quality perinatal care. ${ }^{19-12}$ A systematic review by Liang $e t$ al evaluated 45 studies looking at the mortality from neonatal sepsis in 20 countries, but the setting was limited to developing countries. ${ }^{9}$ Because of heterogeneity in the study population and the risk factors, they did not perform a meta-analysis. ${ }^{9}$ Another systematic review and meta-analysis by Fleischmann-Struzek et al used populationbased data to evaluate the mortality of neonatal sepsis in 12 high-income and middle-income countries but included the older paediatric population. ${ }^{11}$ Given the heterogeneity of available data, as well as a lack of data from all low-income and most 
middle-income countries, the authors mentioned that their estimate for the global burden of neonatal sepsis was considered exploratory. ${ }^{11}$ Bakhuizen et al also carried out a meta-analysis of five studies involving 990 patients to evaluate mortality of neonatal sepsis. ${ }^{12}$ However, it was limited to neonates with a gestation age of less than 34 weeks and/or with birth weight of less than $1500 \mathrm{~g}$. Stronger conclusions could not be made due to limitation on available data. ${ }^{12}$ Therefore, there are current gaps in knowledge about mortality across gestation ages, birth weight and geographical settings. These pieces of information would be necessary to give a better insight about the global burden of mortality following neonatal sepsis.

Classifications of neonatal sepsis are important because the likely causative agents differ between early-onset and late-onset sepsis. ${ }^{413-16}$ Early-onset sepsis is due to transplacental or intrapartum transmission of pathogens from the mother and late-onset sepsis is due to postnatal acquisition of pathogens from community or nosocomial sources. ${ }^{17}{ }^{18}$ Group B streptococcus, Escherichia coli and Listeria monocytogenes are the pathogens frequently responsible for early-onset sepsis, with gram-negative pathogens featuring particularly in preterm infants. ${ }^{19} 20$ Late-onset sepsis is most often attributable to coagulase negative staphylococci and Staphylococcus aureus, especially in the neonatal intensive care unit. ${ }^{21}{ }^{22}$ Herpes simplex virus and enterovirus are more commonly associated with late-onset sepsis, with invasive candidiasis as an emerging cause among neonates treated with broad spectrum antibiotics. ${ }^{23-25}$ Moreover, there are variations in causative pathogens due to differences in geographical locations and settings. ${ }^{26}$

There has been a surge of advances and evolving trends in neonatal sepsis. Guidelines have been established for screening of group B streptococcus in pregnant women and administration of intrapartum antimicrobial prophylaxis to pregnant woman with group B streptococcus. ${ }^{27} 28$ However, this practice can be difficult to implement in lowresource settings where the prevalence of screening is low and the follow-up of women who screen positive is incomplete. ${ }^{29}$ There have been reports on novel preventive strategies such as the administration of fluconazole, lactoferrin, probiotics, anti-staphylococcal monoclonal antibodies, immunoglobulin and granulocyte-macrophage colony-stimulating factors, as well as breastfeeding and establishing measures to minimise healthcare-associated infections. ${ }^{30}$ In addition, there have been new challenges in managing infections due to increasing antibiotic resistance among neonates. ${ }^{3132}$ While medical advancement is commensurate with reduced mortality, new infectious disease trends warrant close study.

There is thus an urgent need at this time for a robust contemporary systematic review and meta-analysis on this topic. In this systematic review, we aim to appraise and summarise the association between neonatal sepsis and death over the past decade. In the meta-analysis, pooled estimates, along with the trends over time, of mortality and case fatality rates of neonatal sepsis will be reported.

\section{METHODOLOGY}

The Preferred Reporting Items for Systematic Reviews and Meta-Analyses 2009 guidelines will be used to conduct this review. ${ }^{33}$

\section{Eligibility criteria}

The eligibility criteria for this systematic review and metaanalysis are as follows:

\section{Inclusion criteria}

- Population: Children less than 90 days old, regardless of gestation and birth weight. In term and post-term newborns, the neonatal period refers to the first 28 days of life, and in preterm newborns, the neonatal period for them is the day of birth, through the expected date of delivery plus 27 days. ${ }^{34}$ Also, serious infections in this population can manifest beyond 28 days of life, including late-onset sepsis associated with group B streptococcus and L. monocytogenes. ${ }^{212}{ }^{22}$ Therefore, to present a complete picture of the disease burden, we choose to study young infants less than 90 days old. If a study includes both paediatric and adult populations, it will only be included if the data pertaining to the paediatric population (less than 90 days old) can be extracted.

- Exposure: Infection of bacterial, viral or fungal origin based on definition or diagnostic criteria for sepsis as determined by the study authors. We chose to include not only bacterial and fungal but also viral infections because young infants can become severely ill from viremic states. ${ }^{45}$

- Outcome: Case fatality rate.

- Design: Randomised controlled trial, cohort study or cross-sectional studies.

- Period: Date of publication between January 2010 and December 2019. We chose this date range with the intention to provide a robust update on a previously published systematic review. ${ }^{11}$ This will also allow the team to focus on case fatality rates over the past decade in neonatal sepsis, given the changes in neonatal sepsis recognition and management. ${ }^{35}$

\section{Exclusion criteria}

- Studies with a primary focus on necrotising enterocolitis, respiratory distress syndrome without a primary sepsis study population, leukaemia or other malignancies.

- Studies with a sample size less than 50 .

- Case reports, animal studies, laboratory studies, publication types of commentaries, letters, books, newsletters, fact sheets, guidelines or editorials.

- Published literature in non-English language.

\section{Information sources}

The literature search will cover the following electronic databases: PubMed, Cochrane Central, Excerpta Medica (Embase) and Web of Science. We also searched the following electronic registries for trial protocols to ensure that there are no completed or ongoing trials evaluating 
the worldwide mortality of neonatal sepsis for both terms and preterm neonates: PROSPERO, ClinicalTrials.gov, International Standard Randomised Controlled Trial Number registry, WHO International Clinical Trials Registry Platform and European Union Clinical Trials Register.

\section{Search strategy}

The search strategy will be developed in consultation with research librarians experienced in systematic reviews and meta-analyses. The search will include all publications from January 2010 to December 2019. Medical Subject Headings (MeSH) are used for PubMed and Cochrane Central. Emtree terms are used for Embase. Topic terms are used for Web of Science. The terms will be exploded as appropriate and their synonyms will be included in the title, abstract and keyword searches.

The full search strategy can be found in online supplementary appendix 1-4. Strategic search terms include: Population-neonate, newborn, infant, baby; Exposure-sepsis, septicaemia, septic shock, pyaemia, endotoxaemia, blood poisoning; Outcome-mortality, fatality, death, demise, survival.

\section{Study records}

Covidence (V.1357.0, Melbourne, VIC, Australia) will be used to facilitate review of articles. After removing duplicates, the studies will be screened for relevance by title and abstract based on the patient, exposure and outcome (PEO) elements detailed above. This will be followed by a full text screening based on the eligibility criteria defined. Each article will be screened by two independent reviewers and any conflicts will be resolved by a third independent reviewer or by discussion. The reasons for excluding any article will be recorded.

\section{Data items}

Two independent reviewers will carry out data extraction using a standardised form consisting of study title, author(s), publication year, geographic origin, study design, enrolment period, sample size, source definitions of neonatal sepsis (clinically defined or culture confirmed), and classification of neonatal sepsis as early or late. Given the lack of consensus on the classification for early-onset versus late-onset sepsis, we will have two separate definitions for early-onset sepsis: less than 7 days and less than 72 hours-this will allow for a comprehensive analysis given current limitations in definitions. ${ }^{17-22}$ We will also document the demographics (eg, gestational age, postnatal age, gender, birth weight), comorbidities, source of infection, causative organisms (ie, bacterial, fungal, parasitic or viral), community-acquired or hospital-acquired infection, blood markers (eg, white cell count, absolute neutrophil count, C-reactive protein, procalcitonin, lactate), number of deaths and time to mortality. Quantitative data will be used for meta-analysis and qualitative data will be used for systematic review.

\section{Outcomes and prioritisation}

The primary outcome of our study is the case fatality rate which is based on the number of deaths within the study population as reported by the study authors. The case fatality rate will be reported by specific timeframes of (1) within 24 hours, (2) 24 hours to less than 7 days and (3) 7 to 30 days from diagnosis of sepsis to occurrence of death. The studies that use numerical tests to measure the case fatality rate will contribute to the meta-analysis, where case fatality rates will be calculated and compared across the following subgroups: gestation age (term vs preterm), birth weight (low birth weight, very low birth weight and extremely low birth weight), early-onset versus late-onset sepsis, community-acquired versus hospital-acquired sepsis and by country's gross national income. ${ }^{36}{ }^{37}$ Young infants 28 days old or less will be presented and analysed on their own as part of a sensitivity analysis in the systematic review and meta-analysis respectively. We will also seek to exclude viral and fungal infections in a sensitivity analysis. The remaining studies will be reviewed systematically and described.

\section{Risk of bias individual studies}

By using the Cochrane Collaboration's tool and ROBINS-I for randomised and non-randomised studies respectively, we will assess each study for selection bias, performance bias, detection bias, attrition bias and reporting bias by assigning a rating of low, high or unclear risk of bias and evaluating the overall strength of the evidence provided by the study. ${ }^{38} 39$

\section{Data synthesis}

Case fatality rates will be calculated for each individual study and presented in forest plots to illustrate the overall effect. Standardised mean differences will be used as the effect measure. This will be determined by a randomeffects approach using the DerSimonian and Laird method. ${ }^{40}$

The original authors will be contacted for missing data. Otherwise, the study will be excluded from meta-analysis but not the systematic review. The systematic review will include a table for the findings of each study and a narrative based on synthesis of this information.

\section{Software to be used}

The software Stata (V.16.0, College Station, Texas, USA) will be used for this meta-analysis.

\section{Meta-bias}

We will assess for publication bias, systematic difference between higher and lower precision studies, as well as any false effects due to poor methodology of the individual studies using a Funnel plot.

\section{Patient and public involvement}

This protocol is developed without patient and public involvement as this is not an interventional study and does not involve patient enrolment. Patients are not invited to comment on the study design and were not 
consulted to develop patient relevant outcomes or interpret the results. Patients are not invited to contribute to the writing or editing of this document for readability or accuracy.

\section{DISCUSSION}

Neonatal sepsis is the most common diagnosis in neonatal intensive care units. ${ }^{14241}$ Despite improvements in the standard of medical care and advances in management strategies, the incidence and mortality rate of neonatal sepsis remains high. ${ }^{42}$ In this systematic review and metaanalysis based on both population and hospital-based studies, we will describe patients' comorbidities, sources of infection and causative organisms of neonatal sepsis, as well as stratify the pool estimates by gestation age, birth weight, onset of sepsis, causative organism, place where infection was acquired and gross national income, ${ }^{36} 37$ in order to identify the predictors of mortality and the factors associated with case fatality in neonatal sepsis. As there are no completed or ongoing trials with this focus, in undertaking this task, we will be able to contribute to a more complete knowledge-based research about the impact of neonatal sepsis, thus facilitating future research and developments to improve care and outcomes for this high-risk population.

\section{Limitations}

There is a lack of consensus-based definitions for neonatal sepsis, unlike in paediatric and adult sepsis. ${ }^{43}$ The neonate's developmental stage and the associated aberrations in host immune response further preclude drawing references from paediatric or adult definitions of sepsis for use in this unique population. Neonatal and young infant sepsis is further compounded by the lack of comprehensive diagnostic testing, particularly in low-resource settings. On one hand, sepsis may not be diagnosed or recognised as a cause of death of those with severe illness, leading to a lower than actual incidence and case fatality rates. On the other hand, deaths may be assumed as sepsis, leading to false labelling and higher than actual incidence and case fatality rates. To address this, we decided to include all studies with sepsis as defined by the authors. However, as a quality measure, we will document if the study team adopted the sepsis definition by Goldstein et al which is a systemic inflammatory response syndrome (SIRS) in the presence of or as a result of a suspected or proven infection. ${ }^{35}$ This definition is relevant and practical to use as age-specific references ranges for vital signs and laboratory variables of SIRS in the neonatal population are clearly stated. Similarly, there is a lack of consensus-based definitions for early-onset and late-onset sepsis. ${ }^{44}$ To handle this data, we will have two separate definitions for early-onset sepsis: less than 7 days and less than 72 hours - this will allow for a comprehensive analysis given current limitations in definitions.

Although using MeSH, Emtree and Topic terms in our search strategy in the various search engines provides a systematic and standardised way of identifying studies in the respective databases, studies may be missed if they were not indexed under the terms used. To address this, we will hand search other systematic reviews and metaanalyses for relevant publications which may have been omitted by the search strategy. Also, the databases are not exhaustive and included only studies published in English language.

Furthermore, by including different study designs in our systematic review and meta-analysis, we anticipate a variable quality of evidence. Mortality in this age group is heavily confounded by other factors which we hope to mitigate by stratifying the following: gestation age, birth weight, early-onset versus late-onset sepsis, communityacquired versus hospital-acquired sepsis and gross national income. ${ }^{36} 37$

This systematic review and meta-analysis of neonatal sepsis and the associated case fatality rate will provide insights about the global healthcare burden of neonatal sepsis. Ultimately, the knowledge gained will provide an update on regions with high fatality rates and facilitate a better understanding of this high-risk population.

\section{Author affiliations}

${ }^{1}$ Emergency Medicine, Sengkang General Hospital, Singapore

${ }^{2}$ Yong Loo Lin School of Medicine, National University of Singapore, Singapore ${ }^{3}$ Department of Pediatrics, Duke University School of Medicine, Durham, North Carolina, USA

${ }^{4}$ Division of Critical Care Medicine, Department of Pediatrics, Duke University School of Medicine, Durham, North Carolina, USA

${ }^{5}$ Department of Paediatrics, KK Women's and Children's Hospital, Singapore ${ }^{6}$ Children's Intensive Care Unit, KK Women's and Children's Hospital, Singapore ${ }^{7}$ Department of Emergency Medicine, KK Women's and Children's Hospital, Singapore

Contributors JHP designed the protocol, as well as drafted and revised the paper. BJY, MYG and STTS designed the protocol and data collection tool, as well as revised the paper. $\mathrm{RG}$ and $\mathrm{CH}$ developed the statistical analysis plan and revised the paper. BT and JHL designed the protocol and data collection tool, as well as revised the paper. S-LC initiated the project, designed the protocol and data collection tool, as well as revised the paper.

Funding This work is funded by National Medical Research Council (Grant Number: CSSSP19may-0006).

Competing interests None declared.

Patient and public involvement Patients and/or the public were not involved in the design, or conduct, or reporting, or dissemination plans of this research.

Patient consent for publication Not required.

Provenance and peer review Not commissioned; externally peer reviewed.

Open access This is an open access article distributed in accordance with the Creative Commons Attribution Non Commercial (CC BY-NC 4.0) license, which permits others to distribute, remix, adapt, build upon this work non-commercially, and license their derivative works on different terms, provided the original work is properly cited, appropriate credit is given, any changes made indicated, and the use is non-commercial. See: http://creativecommons.org/licenses/by-nc/4.0/.

\section{ORCID iDs}

Jen Heng Pek http://orcid.org/0000-0002-8356-7410

Shu-Ling Chong http://orcid.org/0000-0003-4647-0019

\section{REFERENCES}

1 Shane AL, Stoll BJ. Neonatal sepsis: progress towards improved outcomes. J Infect 2014;68 Suppl 1:S24-32. 
2 Liu L, Johnson HL, Cousens S, et al. Global, regional, and national causes of child mortality: an updated systematic analysis for 2010 with time trends since 2000. Lancet 2012;379:2151-61.

3 Waters D, Jawad I, Ahmad A, et al. Aetiology of community-acquired neonatal sepsis in low and middle income countries. J Glob Health 2011;1:154-70.

4 Shane AL, Sánchez PJ, Stoll BJ. Neonatal sepsis. Lancet 2017;390:1770-80.

5 Satar M, Ozlü F. Neonatal sepsis: a continuing disease burden. Turk J Pediatr 2012;54:449-57.

6 INIS Collaborative Group, Brocklehurst P, Farrell B, et al. Treatment of neonatal sepsis with intravenous immune globulin. $N$ Engl J Med 2011;365:1201-11.

7 GBD 2013 Mortality and Causes of Death Collaborators. Global, regional, and national age-sex specific all-cause and causespecific mortality for 240 causes of death, 1990-2013: a systematic analysis for the global burden of disease study 2013. Lancet 2015;385:117-71.

8 Barton L, Hodgman JE, Pavlova Z. Causes of death in the extremely low birth weight infant. Pediatrics 1999;103:446-51.

9 Liang LD, Kotadia N, English L, et al. Predictors of mortality in neonates and infants hospitalized with sepsis or serious infections in developing countries: a systematic review. Front Pediatr 2018;6:277.

10 Investigators of the Delhi Neonatal Infection Study (DeNIS) collaboration. Characterisation and antimicrobial resistance of sepsis pathogens in neonates born in tertiary care centres in Delhi, India: a cohort study. Lancet Glob Health 2016;4:e752-60.

11 Fleischmann-Struzek C, Goldfarb DM, Schlattmann P, et al. The global burden of paediatric and neonatal sepsis: a systematic review. Lancet Respir Med 2018;6:223-30.

12 Bakhuizen SE, de Haan TR, Teune MJ, et al. Meta-Analysis shows that infants who have suffered neonatal sepsis face an increased risk of mortality and severe complications. Acta Paediatr 2014;103:1211-8.

13 Mukhopadhyay S, Puopolo KM. Risk assessment in neonatal early onset sepsis. Semin Perinatol 2012;36:408-15.

14 Camacho-Gonzalez A, Spearman PW, Stoll BJ. Neonatal infectious diseases: evaluation of neonatal sepsis. Pediatr Clin North Am 2013;60:367-89.

15 Cortese F, Scicchitano P, Gesualdo M, et al. Early and late infections in newborns: where do we stand? A review. Pediatr Neonatol 2016;57:265-73.

16 Khan AM, Morris SK, Bhutta ZA. Neonatal and perinatal infections. Pediatr Clin North Am 2017;64:785-98.

17 Rampersaud R, Randis TM, Ratner AJ. Microbiota of the upper and lower genital tract. Semin Fetal Neonatal Med 2012;17:51-7.

18 Read JS, Cannon MJ, Stanberry LR, et al. Prevention of motherto-child transmission of viral infections. Curr Probl Pediatr Adolesc Health Care 2008;38:274-97.

19 Kaufman DA, Coggins SA, Zanelli SA, et al. Congenital cutaneous candidiasis: prompt systemic treatment is associated with improved outcomes in neonates. Clin Infect Dis 2017;64:1387-95.

20 Sgro M, Shah PS, Campbell D, et al. Early-Onset neonatal sepsis: rate and organism pattern between 2003 and 2008. J Perinatol 2011;31:794-8.

21 Bizzarro MJ, Shabanova V, Baltimore RS, et al. Neonatal sepsis 2004-2013: the rise and fall of coagulase-negative staphylococci. $J$ Pediatr 2015;166:1193-9.

22 Vergnano S, Menson E, Smith Z, et al. Characteristics of invasive Staphylococcus aureus in United Kingdom neonatal units. Pediatr Infect Dis J 2011;30:850-4.

23 Thompson C, Whitley R. Neonatal herpes simplex virus infections: where are we now? Adv Exp Med Biol 2011;697:221-30.

24 Modlin JF. Treatment of neonatal enterovirus infections. J Pediatric Infect Dis Soc 2016;5:63-4.

25 Trofa D, Gácser A, Nosanchuk JD. Candida parapsilosis, an emerging fungal pathogen. Clin Microbiol Rev 2008;21:606-25.
26 Vergnano S, Sharland M, Kazembe P, et al. Neonatal sepsis: an international perspective. Arch Dis Child Fetal Neonatal Ed 2005;90:F220-4.

27 Cagno CK, Pettit JM, Weiss BD. Prevention of perinatal group B streptococcal disease: updated CDC guideline. Am Fam Physician 2012;86:59-65.

28 Puopolo KM, Lynfield R, Cummings JJ. Committee on fetus and newborn; Committee on infectious disease. management of infants at risk for group B streptococcal disease. Pediatrics 2019;144:pii :e20191881.

29 World Health Organization. Who recommendation on intrapartum antibiotic administration to women with group B Streptococcus (GBS) colonization for prevention of early neonatal GBS infection. Available: https://extranet.who.int/rhl/topics/preconceptionpregnancy-childbirth-and-postpartum-care/who-recommendationintrapartum-antibiotic-administration-women-group-bstreptococcus-gbs [Accessed 12 May 2020].

30 Shane AL, Stoll BJ. Recent developments and current issues in the epidemiology, diagnosis, and management of bacterial and fungal neonatal sepsis. Am J Perinatol 2013;30:131-41.

31 Laxminarayan R, Matsoso P, Pant S, et al. Access to effective antimicrobials: a worldwide challenge. Lancet 2016;387:168-75.

32 Saha SK, El Arifeen S, Schrag SJ. Aetiology of neonatal infection in South Asia (anisa): an initiative to identify appropriate program priorities to save newborns. Pediatr Infect Dis J 2016;35:S6-8.

33 Liberati A, Altman DG, Tetzlaff J, et al. The PRISMA statement for reporting systematic reviews and meta-analyses of studies that evaluate healthcare interventions: explanation and elaboration. BMJ 2009;339:b2700.

34 U.S. department of health and human services, food and drug administration, center for drug evaluation and research (CDER), center for biologics evaluation and research (CBER). General clinical pharmacology considerations for neonatal studies for drugs and biological products guidance for industry. Available: https://www.fda. gov/media/129532/download [Accessed 19 May 2020].

35 Goldstein B, Giroir B, Randolph A. International consensus conference on pediatric sepsis. International pediatric sepsis consensus conference: definitions for sepsis and organ dysfunction in pediatrics. Pediatr Crit Care Med 2005;6:2-8.

36 Cutland CL, Lackritz EM, Mallett-Moore T, et al. Low birth weight: Case definition \& guidelines for data collection, analysis, and presentation of maternal immunization safety data. Vaccine 2017;35:6492-500

37 The World Bank. World bank country and lending groups. Available: https://datahelpdesk.worldbank.org/knowledgebase/articles/ 906519-world-bank-country-and-lending-groups [Accessed 25 Oct 2019].

38 Higgins JPT, Altman DG, Gøtzsche PC, et al. The Cochrane collaboration's tool for assessing risk of bias in randomised trials. BMJ 2011;343:d5928.

39 Sterne JA, Hernán MA, Reeves BC, et al. ROBINS-I: a tool for assessing risk of bias in non-randomised studies of interventions. BMJ 2016;355:i4919.

40 Sterne J, Bradburn M, Egger M. Meta-Analysis in stata, in systematic reviews in health care: meta-analysis in context. BMJ Publishing 2008:347-69.

41 Clark RH, Bloom BT, Spitzer AR, et al. Reported medication use in the neonatal intensive care unit: data from a large national data set. Pediatrics 2006;117:1979-87.

42 Wynn JL. Defining neonatal sepsis. Curr Opin Pediatr 2016;28:135-40.

43 Wynn JL, Wong HR, Shanley TP, et al. Time for a neonatalspecific consensus definition for sepsis. Pediatr Crit Care Med 2014;15:523-8.

44 Popescu CR, Cavanagh MMM, Tembo B, et al. Neonatal sepsis in low-income countries: epidemiology, diagnosis and prevention. Expert Rev Anti Infect Ther 2020;18:443-52. 